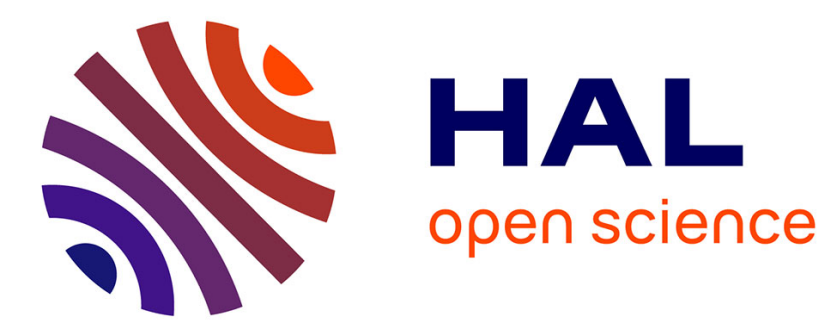

\title{
Non-directed polymers in a random medium
}

\author{
Alex Hansen, Einar Hinrichsen, Stéphane G. Roux
}

\section{To cite this version:}

Alex Hansen, Einar Hinrichsen, Stéphane G. Roux. Non-directed polymers in a random medium. Journal de Physique I, 1993, 3 (7), pp.1569-1584. 10.1051/jp1:1993201 . jpa-00246817

\section{HAL Id: jpa-00246817 https://hal.science/jpa-00246817}

Submitted on 1 Jan 1993

HAL is a multi-disciplinary open access archive for the deposit and dissemination of scientific research documents, whether they are published or not. The documents may come from teaching and research institutions in France or abroad, or from public or private research centers.
L'archive ouverte pluridisciplinaire HAL, est destinée au dépôt et à la diffusion de documents scientifiques de niveau recherche, publiés ou non, émanant des établissements d'enseignement et de recherche français ou étrangers, des laboratoires publics ou privés. 
Classification

Physics Abstracts

$05.40-36.20 \mathrm{E}-68.35 \mathrm{~F}-81.35$

\title{
Non-directed polymers in a random medium
}

\author{
Alex Hansen (1.*), Einar L. Hinrichsen $(1, * *)$ and Stéphane Roux $\left({ }^{2}\right)$ \\ (1) HLRZ der Kernforschungsanlage Jülich. Postfach 1913, D-5170 Jülich 1. Germany \\ (2) LPMMH, Ecole Supérieure de Physique et Chimie Industrielles, URA CNRS 857, 10 rue \\ Vauquelin, F-75231 Paris Cedex 05, France
}

(Received 18 May 1992, revised 8 March 1993, accepted 9 March 1993)

\begin{abstract}
We study an ensemble of polymers that strongly interact with the surrounding medium. One end of the polymers is fixed. but otherwise there are no restrictions on their possible conformations, allowing also self-crossings. We study in this paper by a Flory argument and by numerical methods the statistical properties of these in the low-temperature limit. We find that these properties strongly depend on the disorder of the surrounding medium, in contrast to the directed polymer case.
\end{abstract}

\section{Introduction.}

The statistical mechanics of polymers [1] has been a field of intense study over a number of years now, both due to their interesting properties per se, and as result of their technological importance. The problem of a self-avoiding polymer in a quenched disordered environment has proven to be a highly nontrivial subject within this field, and is not yet completely understood [2]. Fairly recently, Kardar and Zhang [3] have solved a simplified version of this problem, substituting the self-avoidedness of the polymers by assuming that they are directed, i.e. that their conformations are all in a preferred direction without overhangs. Their conclusion is that the behavior of the polymers are governed by a strong-disorder fixed point leading to universal critical behavior different from that of the free self-avoiding polymers. This problem has been studied earlier by a number of authors [4-7], both numerically and analytically. We study in particuliar the low temperature limit of such polymers, whith one end at a fixed position, both analytically and numerically. This problem has also been addressed in references [6] and [7].

Imagine a polymer interacting with a $d$-dimensional random medium through a local interaction energy $\eta(\mathbf{x})$ with no long-range correlations. The polymer also has a bare line tension $\gamma$. The line tension can be caused by entropic and/or elastic effects. The polymer has a

(*) Also at GMCM, URA CNRS 804, Université de Rennes 1, F-35042 Rennes Cedex, France.

(**) Also at: Senter for Industriforskning, Postboks 124, N-0314 Oslo, Norway. 
total length $\ell$. For a given conformation $\mathbf{x}(t)$, where $t$ is a coordinate along the polymer, its total energy is

$$
E\{\mathbf{x}(t)\}=\int_{0}^{p} \mathrm{~d} t^{\prime}\left(\frac{\gamma}{2}\left(\frac{\mathrm{d} \mathbf{x}\left(t^{\prime}\right)}{\mathrm{d} t^{\prime}}\right)^{2}+\eta\left(\mathbf{x}\left(t^{\prime}\right)\right)\right) .
$$

The question is, what is the statistics of the ground state of an ensemble of such polymers, the ground state being characterized by a minimum energy

$$
E_{0}=\min _{\mathbf{v}(t)} E\{\mathbf{x}(t)\} \text {. }
$$

In the special case of directed polymers, the surprising result was found that there is a strongdisorder fixed point in all dimensions - where the random interaction energy $\eta$ dominates in comparison to the line tension $\gamma$ - thus leading to a new universality class [3].

Two universal exponents characterize the fluctuations of the directed polymer, $\chi$ and $\zeta$, and the following scaling relations between fluctuations in energy, $\Delta E$, spatial fluctuations, $R$, and the length of the polymer $\ell$ exist,

and

$$
\Delta E_{0} \sim \ell^{x}
$$

$$
R \sim \ell^{\zeta}
$$

In two dimensions, these exponents are respectively $\chi=1 / 3$ and $\zeta=3 / 2$; in higher dimensions their exact values are not known, but conjectures exist [8].

Fairly recently it has been shown that if the distribution of the random potential $\eta$ has a long power law tail towards negative values, $P(\eta) \sim \eta \rightarrow-\infty|\eta|^{-\beta}$ where $P$ is the cumulative distribution, universality breaks down, and the exponents $\chi$ and $\zeta$ become functions of the disorder $\left(\beta_{-}\right)[9,10]$.

If the disorder becomes infinite, - for example when $1-P(\eta) \sim \eta^{-\beta_{+}}$for large positive $\eta$ and $\beta_{+} \rightarrow 0-$ there is a crossover to directed percolation [11]. The two exponents $\chi$ and $\zeta$ are then related to the transversal and longitudinal correlation length exponents $\nu_{\perp}$ and $\nu_{\|}$by the relations $\chi=1-\frac{1}{\nu_{\|}}$, and $\zeta=\frac{\nu_{\perp}}{\nu_{\|}}$

For the self-avoiding polymers, the situation is still unclear. Le Doussal and Machta [2] argue that there is a strong-disorder fixed point in all dimensions also in this case, by studying hierarchical lattices by Flory-type arguments and by renormalization group analysis.

In the present paper, we make no restrictions on the possible conformations of the polymers except keeping the position of one of their endpoints fixed. In particular, we have not imposed self-avoidedness, nor directedness. By using a Flory argument based on extreme statistics [12] we find that in contrast to the directed polymer case, there is no universal behavior in this case - except in the case of an infinite disorder —, when the problem crosses over to usual percolation. This analysis is supported by extensive numerical studies. Cates and Ball [6] and Nattermann and Renz [7] have also studied this problem, but with results that differ from ours, and which also differ among themselves.

In section 2 we present our statistical analysis based on a Flory argument, and in section 3, we support our analysis by presenting the results of a numerical study of this problem. Section 4 contains a summary and our conclusions.

\section{The statistical analysis.}

We work with a lattice model for the polymer. Whether the lattice constant is the monomer size, or a length scale below which entropic effects dominate, we do not discuss here. However, both in references [6] and [7], the latter length scale is studied. 
Our lattice is a $d$-dimensional hypercubic one. Each node $k$ of the lattice is assigned an interaction energy $\eta_{k}$ drawn from a non-correlated cumulative distribution $P(\eta)$. In this language, the polymer is a self-intersecting path $\mathscr{P}$ starting at the origin of the lattice and having a length of $\ell$ bonds. The total interaction energy of the polymer is $E_{l}\{\mathscr{P}\}=\sum_{k \in \mathscr{P}} \eta_{k}$.

Each time the polymers make a $90^{\circ}$ turn, its total energy picks up a contribution $\gamma$, and for each $180^{\circ}$ a contribution $2 \gamma$ from the line tension.

We divide the possible statistical distributions of the disorder into six different classes : the behavior of the distribution of interaction energies $P(\eta)$ for large positive $\eta$ may be divided into two classes, both behaving either as

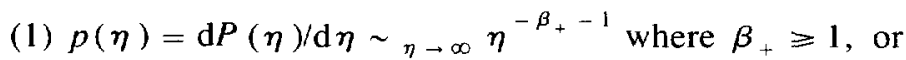

(2) $p(\eta) \sim \sim_{\eta \rightarrow \infty} \eta^{-\beta_{+}^{-1}}$ where $\beta_{+}<1$.

Distributions that approach zero exponentially, or that has a finite cutoff, belong to the first class, as we may sloppily associate them with an infinite $\beta_{+}$. The behavior of $P(\eta)$ for small values of $\eta$ we may divide into three classes. Either we have

(a) a power law behavior $p(\eta) \sim \sim_{--\infty}|\eta|^{-\beta_{-}-1}$, where $\beta_{-} \geqslant 1$,

(b) a power law behavior $p(\eta) \sim \eta_{\eta \rightarrow-}|\eta|^{-\beta_{-}-1}$, where $\beta_{-}<1$,

(c) exponential-type decay. $p(\eta) \sim_{\eta \rightarrow-\infty} \exp \left(-|\eta|^{m}\right)$. For $m=2$ the distribution is Gaussian. Lastly, we may have

(d) a finite cutoff at $\eta_{\ell}$. In this case the behavior of the distribution near this cutoff may be described by an exponent $\alpha, p(\eta) \sim \eta_{\eta} \eta_{\ell}^{+}\left(\eta-\eta_{\ell}\right)^{\alpha-1}$

In contrast to the classification of the different behaviors of the distributions for large $\eta$, we have here singled out the exponential-type distributions and the class of distributions with a finite cutoff, as autonomous classes. The reason for this will be apparent further on.

Ignoring the line tension, $\gamma$, the ground state conformation of a polymer that has no end attached to a fixed point will be completely curled up between the two neighboring nodes, $i$ and $j$, whose sum of interaction energies $\eta_{1}+\eta_{1}$ is the smallest possible.

If we keep one end of the polymer fixed - still ignoring the line tension - the polymer will take on what is called a «tadpole » configuration in reference [7]: it will stretch out to a neighboring pair of nodes, $i$ and $j$, whose sum of interaction energies, $\eta_{1}+\eta_{j}$, is so low that it is energetically favorable for the polymer to curl up between these two nodes for what is left of its total length. The conformation of the polymer up to this pair of nodes contains no self crossings, even when the interaction energy is attractive and unbounded. We illustrate this in figure 1. In figure 2 we show the different positions of the « head $»$ of the tadpole configuration as a function of the length of the polymer, corresponding to the three configurations shown in figure 1 , and finally in figure 3 we show the corresponding figure of polymers starting at all nodes. This shows how the different possible positions of the "heads " of the tadople configurations starting at different nodes converge with increasing length of the polymers.

The fixed starting point of a polymer of length $\ell$ is at a. It stretches out to a point $b$ at which it curles up. The distance between points $a$ and $b$ is $r$. This distance is covered by a portion $\ell_{\mathrm{a}}$ of the entire polymer. The rest of the polymer $\ell_{\mathrm{b}}=\ell-\ell_{\mathrm{a}}$ is curled up in the « head" of the tadpole at $b$. The total energy of the polymer consists of two terms : one which is the sum of interaction energies along the path of length $\ell_{\mathrm{a}}$ taken by the polymer from point a to $\mathrm{b}, \mathrm{d}_{\mathrm{a}}$

$$
E_{\mathrm{d}}=\sum_{, \in 1} \eta_{\imath}
$$




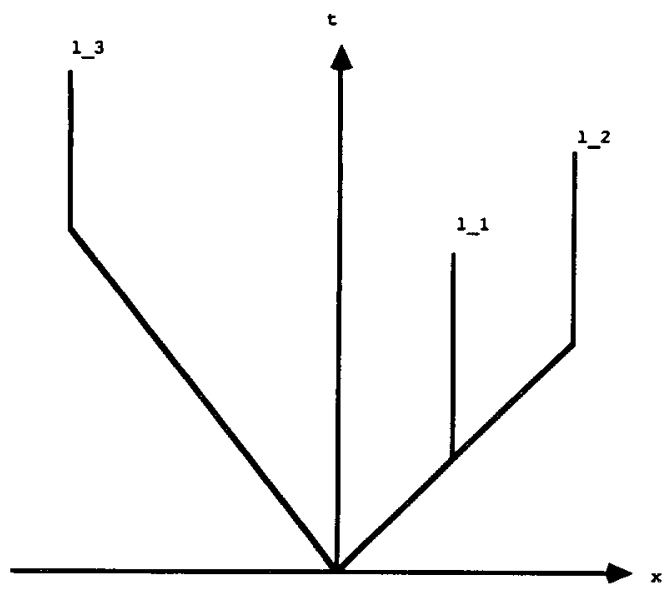

Fig. 1.

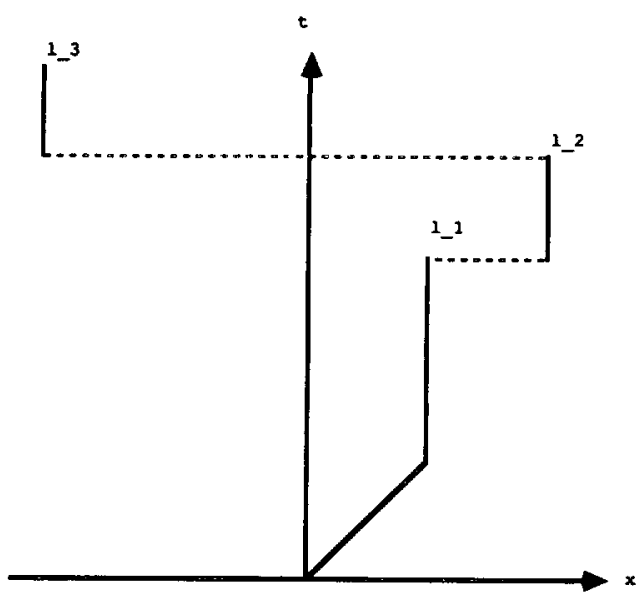

Fig. 2.

Fig. 1. - The three curves $x(t)$ show the optimal configurations for a polymer of length $\ell$ less than or equal to $\ell_{1}, \ell_{1}<\ell<\ell_{2}$, and $\ell_{2}<\ell \leqslant \ell_{3}$ starting at the origin in a one-dimensional lattice. Here $x(t)$ should be interpreted as the spatial position of the $t$-th monomer from the endpoint at the origin.

Fig. 2. - The curve $x(t)$ signifies the different positions of the " head $»$ of the tadpole configuration as a function of the length of the polymer, corresponding to the three configurations shown in figure 1.

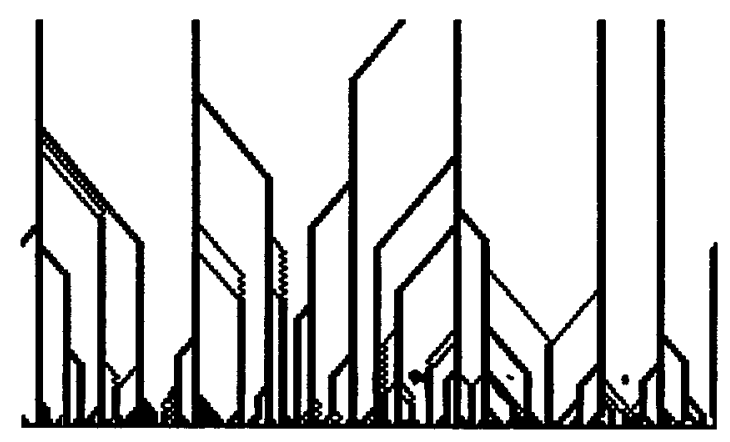

Fig. 3. - This figure is a generalization of figure 2 to polymers starting at all nodes in the onedimensional lattice. We illustrate here how the density of « heads » diminish as a function of the length of the polymer. The mean distance between these "heads" defines a correlation length.

while the other part is the energy of the curled-up part of the polymer,

$$
E_{\mathrm{b}}=\ell_{\mathrm{b}} \eta_{\mathrm{b}},
$$

where $\eta_{\mathrm{b}}$ is the interaction energy of the neighboring nodes at $b$.

The Flory argument consists - as usual - in estimating separately the two interaction energies, $E_{\mathrm{a}}$ and $E_{\mathrm{b}}$, as a function of the distance $r$ between the two end points, a and $\mathrm{b}$, of the polymer. We then minimize the total energy $E=E_{\mathrm{a}}+E_{\mathrm{b}}$ with respect to $r$.

The interaction energy of the tail of the tadpole configuration, $E_{\mathrm{a}}$, may be written

$$
E_{\mathrm{a}}=\ell_{\mathrm{d}}\langle\eta\rangle_{\mathscr{P}_{\mathrm{a}}},
$$


where $\langle\eta\rangle_{\mathscr{F}}$ is the average interaction energy along the path $\mathfrak{T}_{\mathrm{a}}$. The length of the path $\mathfrak{T}_{\mathrm{a}}$ scales as $r^{\delta}$, where $\delta \geqslant 1$. We note that $E_{\mathrm{a}}$ increase with increasing $\delta$, and thus make the assumption that $\delta=1$, so that $r$ is proportional to $\boldsymbol{\ell}_{\mathrm{a}}$, the length of the path $\mathfrak{I}_{\mathrm{a}}$ - for simplicity we set $\ell_{\mathrm{a}}=r$. The second assumption we make is that the correlated average $\langle\eta\rangle_{\mathbb{S}_{\mathrm{A}}}$ may be substituted by an uncorrelated average over $\ell_{\mathrm{a}}$ elements. Thus, we may write

$$
E_{\mathrm{a}}=r\langle\eta\rangle,
$$

The interaction energy of the «head » of the polymer we estimate as

$$
E_{\mathrm{b}}=\ell_{\mathrm{b}} \eta_{\min (1)}=(\ell-r) \eta_{\min (r)},
$$

where $\eta_{\min (1)}$ is the minimum $\eta_{1}+\eta_{1}$ over all neighboring nodes $i$ and $j$ within a radius $r$ of the start point of the polymer.

The total interaction energy is thus

$$
E(r)=r\langle\eta\rangle,+(\ell-r) \eta_{\min (1)} .
$$

The ground state is thus given by

$$
E_{0}=\min E(r)
$$

The ground state energy scales with the polymer length $\ell$ as

$$
E_{0} \sim \ell^{\mu}
$$

The exponent $\mu \geqslant 1$. This is so since adding a constant $\eta_{0}$ to each value of $\eta$ does not change the relative energies of the various conformations of the polymers, as it only adds a constant term $\ell \eta_{0}$ to the total energy. Thus, there will always be a term present with scaling exponent equal to one - and depending on the distribution of $\eta$, it may be the leading term or a correction term. We may subtract in equation (11) the leading term. What is left are the fluctuations.

$$
\Delta E_{0}(\ell)=E_{0}(\ell)-\ell^{\mu} \lim _{\ell^{\prime} \rightarrow \infty} E_{0}\left(\ell^{\prime}\right) / \ell^{\prime \mu}
$$

Let us now return to the situation where the line tension $\gamma$ is not zero. Assuming a conformation as that of equation (5), the part of the polymer that is « curled up » at b picks up an additional energy $2 \gamma(\ell-r)$. The number of turns the polymer makes along the path leading from its fixed endpoint to the minimal-energy nodes we expect to be proportional to $r$. Thus, from this part of the polymer there is an additional energy $C_{\gamma_{l}}$. Equation (10). then, is changed into

$$
E(r)=r\langle\eta\rangle(r)+(C-2) \gamma r+(\ell-r) \eta_{\min (r)}+2 \gamma \ell .
$$

The last term of this equation may be removed simply by rescaling the interaction energies. Also the second additional term, $(C-2) \gamma r$ does not change the behavior of the polymers. We will return to this point momentarily.

The average interaction energy $\langle\eta\rangle$, used in equation (8) is

$$
\langle\eta\rangle_{1}=\int_{\eta_{\min (s)}}^{\eta_{\max (a)}} \eta p(\eta) \mathrm{d} \eta \sim \begin{cases}\text { constant, } & \text { in case }(1 \mathrm{y}), \\ r^{1 / \beta_{+}-1}, & \text { in case }(2 \mathrm{y}), \\ -r^{1 / \beta_{-}-1}, & \text { in case }(1 \mathrm{~b}), \\ \pm r^{1 / \beta_{ \pm}-1}, & \text { in case }(2 \mathrm{~b}),\end{cases}
$$


where 1 and 2 refer to the two classes of statistical distributions for large values of $\eta$, while $b$ and $y=a, c$, or $d$ refer to the four classes of asymptotic distributions for small values of $\eta$. The exponent

$$
\beta_{ \pm}= \begin{cases}\beta_{+}, & \text {if } \beta_{+}<\beta_{-} \\ \beta_{-}, & \text {if } \beta_{+}>\beta_{-}\end{cases}
$$

We have here used that the expected maximum or minimum value of $r \eta$-values drawn from the distribution $P(\eta)$ behaves as [12] $P\left(\eta_{\max (1)}\right)=1-\frac{1}{r}$, and $P\left(\eta_{\min (i)}\right)=\frac{1}{r}$

Returning to equation (14), we see that in cases ( $1 \mathrm{y})$, the only effect of the additional term $(C-2) \gamma r$ is to change the prefactor in equation (8), $1 \rightarrow 1+(C-2) \gamma$, thus having no influence on the scaling behavior of the polymers. In the present formulation of the problem, the interaction energy of each site can be redefined in order to incorporate the additional energy cost due to the line tension since the latter is simply dictated by the local geometry of the medium. Thus, up to this redefinition. which will not affect the scaling of the interaction energies, the line tension does not play any role in the phase diagrams we discuss in the following. In the other cases, the term $r\langle\eta\rangle$, will dominate compared to the term $(C-2) \gamma r$, so that it may simply be ignored. Thus, in both cases, it is the disorder which determines the behavior of the polymers - not the line tension. Note in particular that this argument is independent of the dimension $d$.

We now have to estimate the smallest pair of interaction energies we expect to find. There are four different cases to discuss, corresponding to the three classes (a), (b), (c) and (d) defined above. In cases (a) and (b), if there is a power law tail towards large negative values of $\eta, p(\eta) \sim \eta \rightarrow-\infty|\eta|^{-\beta_{-}-1}$, then the distribution for the sum of two independently chosen $\eta$ will be the same. This is also the case of the exponential-type distributions of class (c). In the last case, (d), we add a constant $\eta_{0}$ to the interaction energies, so that the lower cutoff $\eta_{\ell}$ is at 0 . Then, the behavior of the distribution near 0 behaves as $p(\eta) \sim \eta \rightarrow 0^{+} \eta^{\alpha-1}$ The sum of two such numbers are then distributed according to

$$
p^{\prime}(\eta)=\int_{\eta} \mathrm{d} \eta^{\prime} p\left(\eta^{\prime}\right) p\left(\eta-\eta^{\prime}\right) \sim \eta^{2 \alpha-1}
$$

In the same way as we have estimated the largest value above, we estimate the smallest sum of two $\eta$,

$$
P^{\prime}\left(\eta_{\min (1)}\right)=r^{-d}
$$

This gives

$$
\eta_{\min (s)} \sim \begin{cases}r^{d / \beta_{-}}, & \text {in case a) } \\ r^{d / \beta_{-}}, & \text {in case b) } \\ (\ln r)^{1 / m} & \text { in case c) } \\ r^{-d / 2 \alpha}, & \text { in case d) }\end{cases}
$$

Inserting equations (14) and (18) in equation (10), we minimize with respect to $r$, thus determining $r$ as a function of $\ell$. Each of the eight different behaviors for small and large values of $\eta$ has to be analysed separately, giving rise to three phase diagrams, one based on cases (1a), (1b), (2a), and (2b); a second one based on cases (1c) and (2c); and a third phase diagram based on cases (1d) and (2d). In the minimization, two situations can arise : either the minimum corresponds to $\partial E(r) / \partial r=0$ if $r<\ell$, or the minimum is reached for the maximum 
extension the polymer can achieve : $r=\ell$, the minimum of the function $E(r)$ being located in the unphysical regior $r>\mathbb{P}$. The first phase diagram, which is shown in figure 4 , is characterized by five phases, A, B, C, D and E. They are characterized by

$$
r \sim \begin{cases}\text { constant }, & \text { in phase A ; } \\ \ell^{\beta} \beta_{-}\left(\beta_{-}-d \beta_{+}\right) & \text {in phase B ; } \\ \ell, & \text { in phase C ; } \\ \ell, & \text { in phase D; } \\ \ell, & \text { in phase E . }\end{cases}
$$

In phase $\mathrm{A}$, the polymer is collapsed. The first order line separating phases $\mathrm{A}$ and $\mathrm{C}$ phase is given by $\beta_{-}=\beta_{+}$for $\beta_{+} \leqslant 1$, and $\beta_{-}=1$ for $\beta_{+}>1$; the first order line separating phases $\mathrm{A}$ and $\mathrm{D}$ is given by $\beta_{-}=d$ for $\beta_{+}>1$; the first order line separating phases $\mathrm{A}$ and $\mathrm{E}$ is given by $\beta_{-}=d \beta_{+}$for $\beta_{+} \leqslant 1$; the first order line separating phases $\mathrm{B}$ and $\mathrm{E}$ is given by $\beta_{-}=d \beta_{+} /\left(1-\beta_{+}\right)$; and the second order line separating phases $\mathrm{D}$ and $\mathrm{E}$ is given by $\beta_{+}=1$ for $\beta_{-}>d$.

By using the terminology « first order » and « second order » lines, we distinguish between a discontinuity in the exponent values, for a discontinuity in their derivative with respect to the parameters $\beta_{+}$and $\beta_{-}$.

The second phase diagram, figure 5 , is based on cases (1c) and (25c). There are three phases, F, G and $\mathrm{H}$,

$$
r \sim \begin{cases}\ell^{\beta} \cdot(\log \ell)^{1 / m-1}, & \text { in phase } F ; \\ \ell, & \text { in phase } G ; \\ \ell, & \text { in phase } \mathrm{H} .\end{cases}
$$

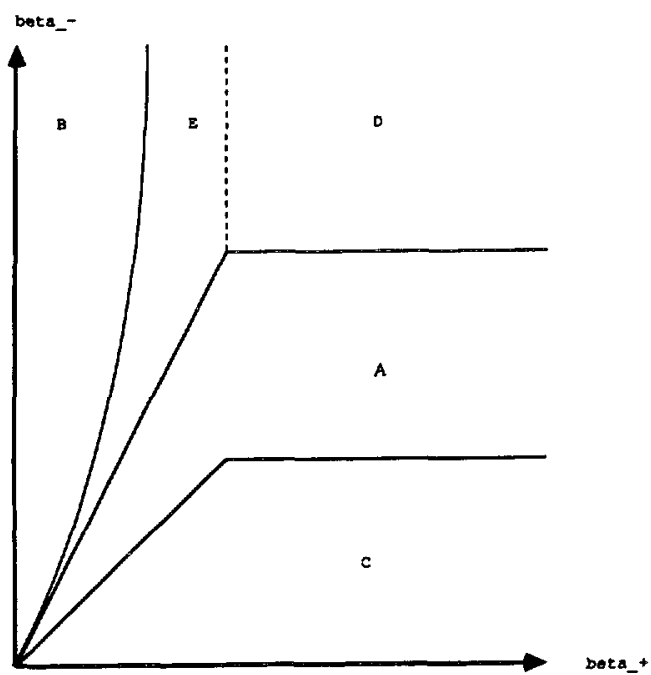

Fig. 4.

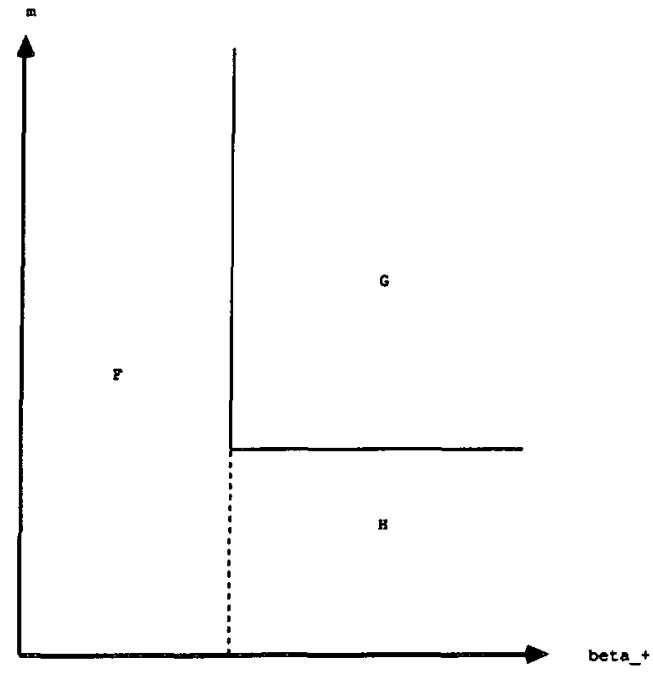

Fig. 5.

Fig. 4. - Phase diagram for the asymptotic behavior of the distribution $p(\eta)$ when $p(\eta) \sim_{\eta \rightarrow \infty} \eta^{\beta_{+}-1}$ (cases 1 and 2), and $p(\eta) \sim \eta \rightarrow-\infty|\eta|^{\beta_{-}-1}$ (cases a and b).

Fig. 5. - Phase diagram for the asymptotic behavior of the distribution $p(\eta)$ when $p(\eta) \sim \eta_{\eta \infty} \eta^{\beta_{+}-1}$ (cases 1 and 2), and $p(\eta) \sim \eta \rightarrow-\infty \exp \left(-|\eta|^{m}\right)$ (case $\mathfrak{c}$ ). 
They are separated by the first order lines $\beta_{+}=1$ for $m>1$, and $m=1$ for $\beta_{+}>1$, and the second order line $\beta_{+}=1$ for $0<m<1$.

The third phase diagram is based on cases (1d) and (2d). This is shown in figure 6. Here, there are only two phases, I and J, characterized by

$$
r \sim \begin{cases}\ell^{2 \alpha /(2 \alpha+d)}, & \text { in phase } \mathrm{I} ; \\ \ell^{2 \alpha \beta_{+} /\left(2 \alpha+d \beta_{-}\right)}, & \text {in phase } \mathrm{J} .\end{cases}
$$

The second order line separating phases $\mathrm{I}$ and $\mathrm{J}$ is given by $\beta_{+}=1$.

Equations (20), (21) and (22) show that there is no universal value for the exponent $\zeta$, defined through the equations $r \sim \ell^{\zeta}$. We may also determine the scaling exponents of the ground state energy, $E_{0} \sim \ell^{\mu}$, and the fluctuations, $\Delta E_{0} \sim \ell^{\chi}$ using equation (13). We give in table I the values of $\mu$ and $\chi$ together with $\zeta$ for all the seven different phases that may be encountered. When substituting $r=r(\ell)$ as given in equation (20), equations (21) and (22) in

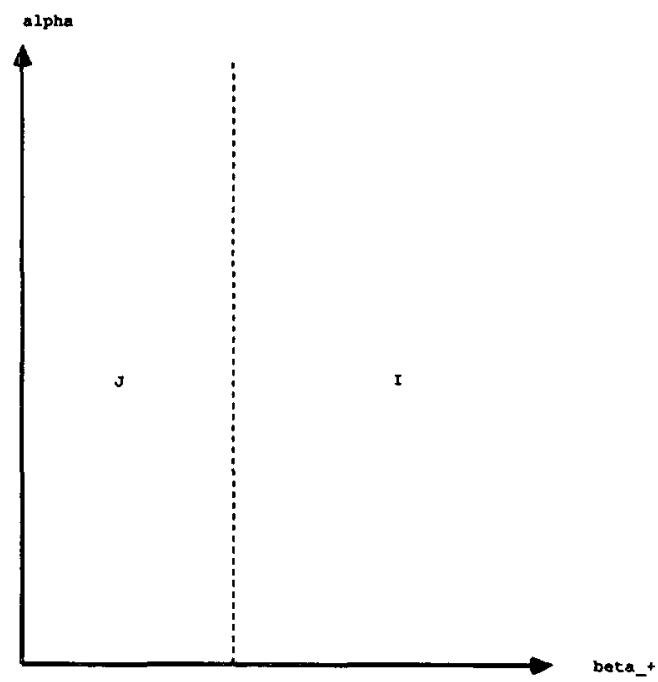

Fig. 6. - Phase diagram for the asymptotic behavior of the distribution $p(\eta)$ when $p(\eta) \sim \eta \rightarrow \infty \eta^{\beta_{+}-1}$ (cases 1 and 2), and $p(\eta) \sim \eta \rightarrow \eta \downarrow\left|\eta-\eta_{\ell}\right|^{\alpha-1}$ (case d).

Table I. - The exponents $\zeta, \chi$, and $\mu$ defined by $r \sim \ell^{\zeta}, \Delta E_{0} \sim \ell^{\chi}$ and $E_{0} \sim \ell^{\mu}$ for the different phases A to H, shown in figures 4, 5 and 6 .

\begin{tabular}{|c|c|c|c|}
\hline Phase & $\zeta$ & $\chi$ & $\mu$ \\
\hline$A$ & 0 & 0 & 1 \\
$B$ & $\beta_{-} \beta_{+} /\left(\beta_{-}-d \beta_{+}\right)$ & $\beta_{-} /\left(\beta_{-}-d \beta_{+}\right)$ & $\beta_{-} /\left(\beta_{-}-d \beta_{+}\right)$ \\
$C$ & 1 & $1 / \beta_{-}$ & $1+\left(d / \beta_{-}\right)$ \\
$D$ & 1 & 1 & $1+\left(d / \beta_{-}\right)$ \\
$E$ & 1 & $1 / \beta_{+}$ & $1+\left(d / \beta_{-}\right)$ \\
$F$ & $\beta_{+}+\log . \operatorname{corr}$. & $1+\log$. corr. & $1+\log$. corr. \\
$G$ & 1 & $1+\log$. corr. & $1+\log$ corr. \\
$H$ & 1 & 1 & 1 \\
$I$ & $2 \alpha /(2 \alpha+d)$ & $2 \alpha /(2 \alpha+d)$ & 1 \\
$J$ & $2 \alpha \beta_{+} /\left(2 \alpha+d \beta_{+}\right)$ & $2 \alpha /\left(2 \alpha+\right.$ det $\left.t a_{+}\right)$ & 1 \\
\hline
\end{tabular}


equation (10), for some phases we find a $\mu<1$. In light of the discussion following equation (12), we set $\chi$ equal to this value, and substitute $\mu=1$. It should be noted that the relation [13] $\chi=2 \zeta-1$ is not valid as was also pointed out in reference [7]. The underlying assumption resulting in this relation is that the fluctuations $\Delta E_{0}$ become analytic for large values of $\ell$ and $r-$ which is clearly not the case here.

In references [6] and [7] only the Gaussian potential is treated, i.e. case (1c), with $m=2$ in our classification scheme. We predict in this case $r \sim \frac{\ell}{\sqrt{\log \ell}}$. Cates and Ball [6] find that $r \sim \frac{\ell}{\log \ell}$, while Nattermann and Renz [7] find $r \sim \frac{\ell^{\prime / \log \ell}}{(\log \ell)^{3 / 4}}$. Both of these calculations are based on Flory-type arguments.

In the case when the disorder becomes « infinite ", the problem crosses over to a percolation one. We return to equation (2), written in terms of the lattice,

$$
E_{0}=\min _{\mathbf{x}(t)} \sum_{h \in \mathbf{x}(t)} \eta_{k}
$$

In the limit of « infinite » disorder it becomes

$$
E_{0}=\min _{\mathbf{v}(t)} \max _{k \in \mathbf{x}(t)} \eta_{k}
$$

where $P^{-1}\left(E_{0} / \ell\right) \rightarrow p_{\mathrm{c}}$ as $\ell \rightarrow \infty$, where $p_{\mathrm{c}}$ is the percolation threshold of the lattice [11]. In this limit, the exponent $\zeta$ becomes $d_{\mathrm{m} \text { n }}$ - the chemical distance exponent [14], while $\chi=1-\frac{1}{\nu d_{\min }}$, where $\nu$ is the percolation correlation length exponent. We note that in this limit, the extreme-statistics arguments leading to the determination of $\chi$ and $\zeta$ are no longer valid, as there is no competition between the different factors of the total energy, equations (7) and (9).

\section{Numerical results.}

We now turn to the results of our numerical studies of this problem. We generated an ensemble of polymers in one and two dimensions by treating them as directed polymers in a space with one extra dimension. This extra dimension we call the $t$-direction, and signifies the numbering of the monomers from one end of the polymer. The position of the $t$-th monomer is characterized by $(\mathbf{x}, t)$ in this enlarged space. This is illustrated in figures 1 and 2 . The interaction energies $\eta$ are functions only of the spatial coordinates $\mathbf{x}-$ thus, the problem treated this way is in reality one of directed polymers with quenched disorder. We imagine "growing " the polymer by adding layer after layer in the $t$-direction, searching for the minimal energy ignoring the line tension. We define for each lattice site $\mathbf{x}=\mathbf{k}$ an energy $E(\mathbf{k}, t)$. At $t=0$, we set all $E(\mathbf{k}, 0)$ to a very large positive number, except for $\mathbf{k}=0$, the origin. We then assign interaction energies $\eta_{k}$ to each node, and update according to the rule

$$
E(\mathbf{k}, t+1)=\min _{n n(\mathbf{k})}\left(E(n n(\mathbf{k}), t)+\eta_{\mathbf{k}}\right),
$$

where $n n(\mathbf{k})$ refers to the nearest neighbor nodes to node $\mathbf{k}$. When the polymer has reached its desired length $\ell$, the minimum $E(k, \ell)$ is found. This gives us the minimum energy for a polymer with only one end fixed. We may also study closed polymers by reading off $E(\mathbf{k}, \ell)$ at the same node at which we started growing the polymer. In figure 7 we show the 


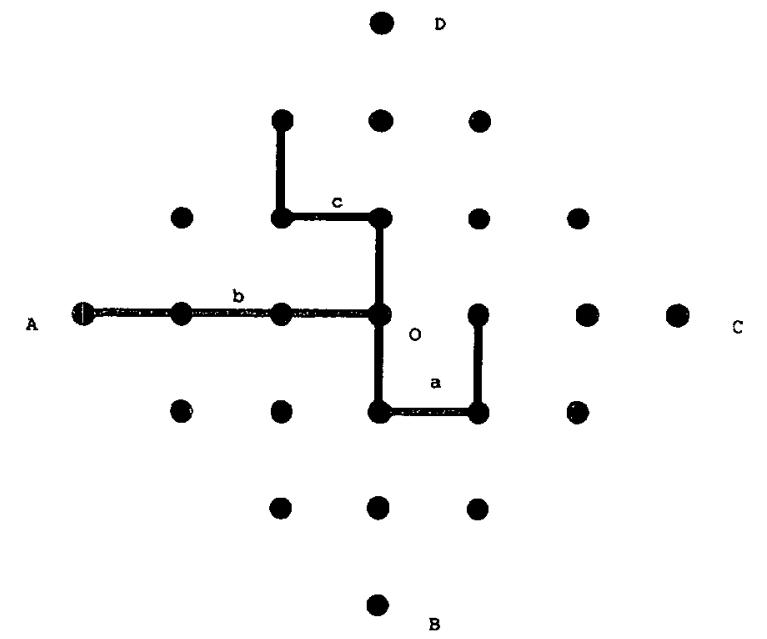

Fig. 7. - The geometry of the two-dimensional lattice we have used. Three possible polymer configurations named $a, b$ and $c$ are shown.

geometry of the lattice used for our two-dimensional studies. A polymer of length $\ell=5$ may reach as far as the diagonals joining pairwise the four corners marked $A, B, C$ and $D$. Polymers that stretch from the center node $O$ to either of the four outer diagonals $A B, B C$, $\mathrm{CD}$ or DA have no overhangs and are thus directed. For ensembles of such polymers we expect [8] $\chi=1 / 3$ and $\zeta=3 / 2$, as long as the underlying distribution of local interaction energies does not have a power law tail $p(\eta) \sim \eta \rightarrow-\infty|\eta|^{-\beta_{-}-1}-$ i.e. is of type (a) or (b) - with an exponent $\beta_{-}<6[9,10]$. We also note that for polymers ending at either of the four corners $A$, $\mathrm{B}, \mathrm{C}$ or $\mathrm{D}$, the polymers is fully stretched, and only one conformation is possible. Thus, the total energy of such polymers is just the sum of independent random numbers, and we expect to find that their total energy either obey Gaussian statistics - or if the underlying interaction energy distribution $p(\eta)$ has long power law tails towards $\pm \infty$, we expect Lévy distributions. These special cases allow us to to check the accuracy of our numerical results.

Using the Cray Y/MP-832 at HLRZ, Jülich, we generated a number of realizations for each length of the polymers ranging from $\ell=8$ to $\ell=1150$ in $d=1$ dimension, and $\ell$ ranging from 8 to 406 in two dimensions, as shown in table II. We studied one distribution in one dimension, and in two dimensions five different distributions of local interactions energies, $\eta$,

(a) the cumulative distribution $P(\eta)=\eta^{1 / 2}$ for $0<\eta<1$, i.e. a type (1d) distribution ;

(b) the cumulative distribution $P(\eta)=\eta$ for $0<\eta<1$; i.e. the interaction energies are uniformly distributed on the unit interval. This distribution is also of type (1d). It is furthermore the distribution we used for the one-dimensional case ;

(c) the cumulative distribution $P(\eta)=\eta^{2}$ for $0<\eta<1$, again a type (1d) distribution ;

(d) The cumulative distribution $P(\eta)=1-\eta^{-1}$ for $1<\eta<\infty$. For $\eta$ near one, this distribution behaves as $P(\eta)=(\eta-1)$. Thus, $\beta_{+}=1$ and $\alpha=1$, placing it on the border line between $(1 \mathrm{~d})$ and $(2 \mathrm{~d})$;

(e) The cumulative distribution $P(\eta)=|\eta|^{-1}$ for $-\infty<\eta<-1$. This is again a borderline distribution between types (1a) and (1b), as $\beta_{-}=1$.

In order to give an impression of the quality of our numerical results, we show in figures 8 and $9 \Delta E_{0}$ as a function of $\ell$ for distribution (b) for polymers in two dimensions ending at either the four «Gaussian points » $A, B, C$ and $D$ (conformations such as the one marked $b$ in Fig. 7) and for polymers ending at the outer diagonal, $A B, B C, C D$ and $D A$, forcing the 
Table II. - Number of realizations studied in one and two dimensions for different lengths $\ell$ of the polymers.

\begin{tabular}{|c|c|c|}
\hline length $l$ & Realiz. in $d=1$ & Realiz. in $d=2$ \\
\hline 8 & 100000 & 100000 \\
12 & 100000 & 100000 \\
18 & 100000 & 100000 \\
24 & 100000 & 50000 \\
34 & 100000 & 30000 \\
50 & 100000 & 10000 \\
70 & 100000 & 7200 \\
100 & 50000 & 2500 \\
142 & 50000 & 1000 \\
202 & 50000 & 500 \\
286 & 30000 & 200 \\
406 & 30000 & 100 \\
574 & 30000 & \\
814 & 10000 & \\
1150 & 10000 & \\
\hline
\end{tabular}

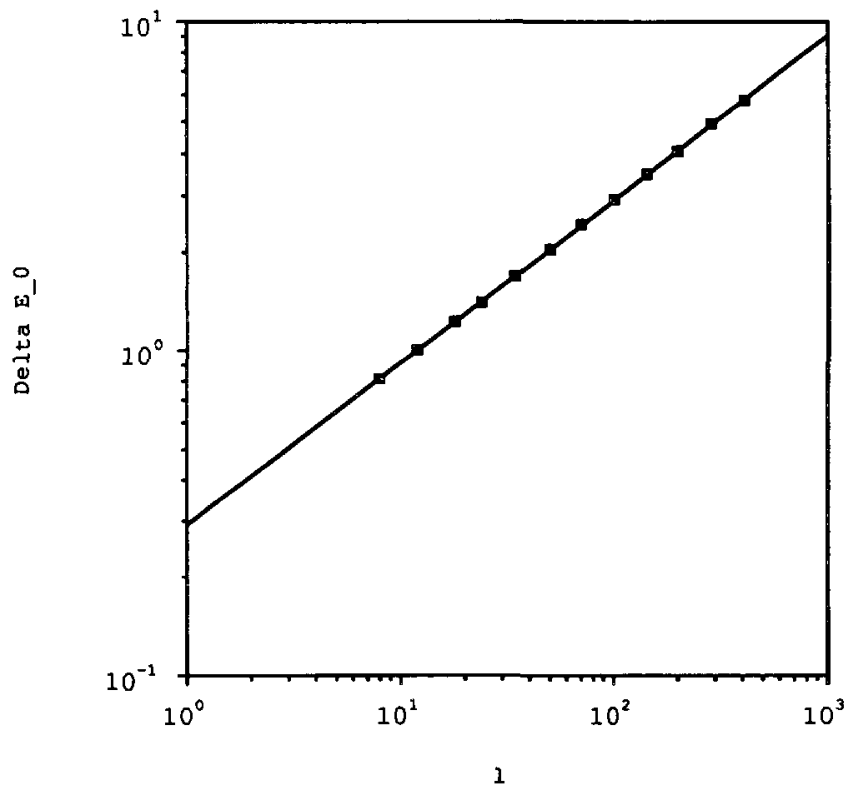

Fig. 8. $-\Delta E_{0}$ as a function of $\ell$ for polymers ending at either of the extreme points $A, B, C$ and $D$ with a uniform distribution of local interaction energies $\eta$ between zero and one (distribution e). The slope is $0.50 \pm 0.01$.

polymers to be directed (conformations such as the one marked $c$ in Fig. 7). The expected slopes are respectively $1 / 2$ for the Gaussian case and $1 / 3$ for the directed polymer case. We find $0.50 \pm 0.01$ for the first case, and $0.33 \pm 0.01$ for the second one.

In table III we present our measurements of the three exponents $\zeta . \chi$ and $\mu$ compared to the predictions of the Flory theory presented in section two. All exponents are measured two 


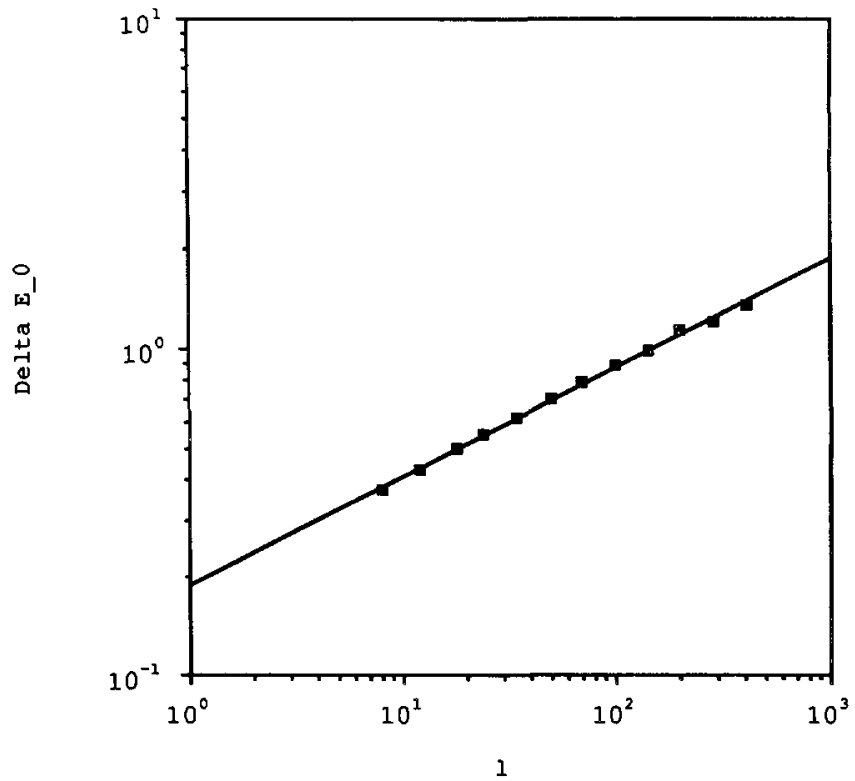

Fig. 9. $-\Delta E_{0}$ as a function of $\ell$ for polymers ending somewhere either of the extreme diagonals $A B$, BC. CD and DA with a uniform distribution of local interaction energies $\eta$ between zero and one (distribution e). The slope is $0.33 \pm 0.01$.

Table III. - Numerically measured and Flory values for the three exponents $\zeta, \chi$ and $\mu$ for the different distributions for local interaction energy we studied, in both one and two dimensions. In the column $\mu_{\text {Flory }}$ we have given in parantes the values found by minimizing the energy expression (Eq. (10)). These values are all less than one, and have therefore been substituted by the physical value one (marked by $a^{*}$ ). However, in our computer simulations we see these unphysical values for $\mu$. The values for $\zeta_{\text {Flory }}, \chi_{\text {Flory }}$ and $\mu_{\text {Flory }}$ in the last row are those of phases $\mathrm{A}$ and $\mathrm{C}$, as the distribution (e) sits right on the line separating the two. The accuracy of the exponents $\chi_{\text {meas }}$ nor $\mu_{\text {meas }}$ in this case are only rough estimates.

\begin{tabular}{|c|c|c|c|c|c|c|c|}
\hline Dim. & Distribution & $\zeta_{\text {meas. }}$ & $\zeta_{\text {Flory }}$ & $\chi_{\text {meas. }}$ & $\chi_{\text {Flory }}$ & $\mu_{\text {meas. }}$ & $\mu_{\text {Flory }}$ \\
\hline 1 & $\mathrm{~b}$ & 0.66 & $2 / 3$ & 0.67 & $2 / 3$ & 0.68 & $(2 / 3) 1^{*}$ \\
2 & $\mathrm{a}$ & 0.28 & $1 / 3$ & 0.29 & $1 / 3$ & 0.4 & $(1 / 3) 1^{*}$ \\
2 & $\mathrm{~b}$ & 0.44 & $1 / 2$ & 0.45 & $1 / 2$ & 0.52 & $(1 / 2) 1^{*}$ \\
2 & $\mathrm{c}$ & 0.58 & $2 / 3$ & 0.66 & $2 / 3$ & 0.7 & $(2 / 3) 1^{*}$ \\
2 & $\mathrm{~d}$ & 0.50 & $1 / 2$ & 0.36 & $1 / 2$ & 0.98 & $(1 / 2) 1^{*}$ \\
2 & $\mathrm{e}$ & 0.96 & 1 & $\sim 3$ & 1 & $\sim 3$ & 3 \\
\hline
\end{tabular}

ways : by either an ensemble of open polymers, i.e. polymers with one free end, or by an ensemble of closed polymers, i.e. polymers forming a ring. The radius $r$ we measured either as their largest spread, i.e. the distance from starting point to any part of the polymer, for both the open and closed polymers, or as the average distance between start point and endpoint for the open ones only. In figures 10 and 11 we show $r$ as a function of $\ell$ gotten by measuring the largest spread of both the open and the closed polymers, and by end-to-end distance of the open polymers for distribution (b) in one and two dimensions. Likewise, in figures 12 and 13 we show the scaling of the ground state energy, $E_{0}$ and the fluctuations, $\Delta E_{0}$ with respect to $\ell$ 


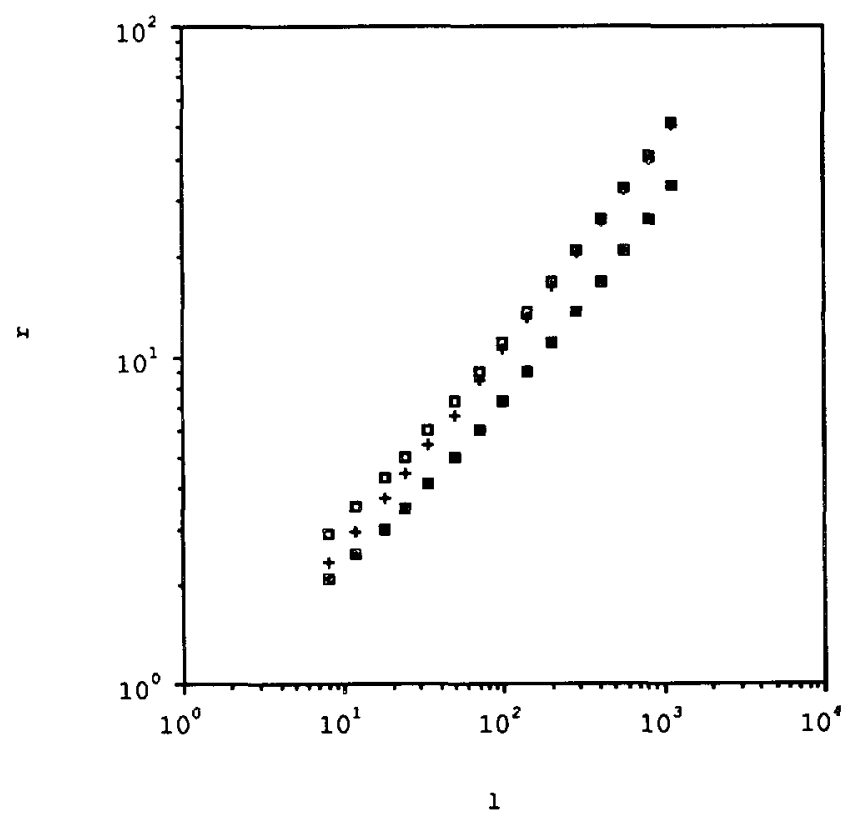

Fig. 10. $-r$ as a function of $\ell$ measured by the largest spread of either closed ( $\square$ ) or open polymers ( $\square$ ), or as the end-to-end distance for open polymers $(+)$ in one dimension and a uniform local interaction energy distribution between zero and one (b).

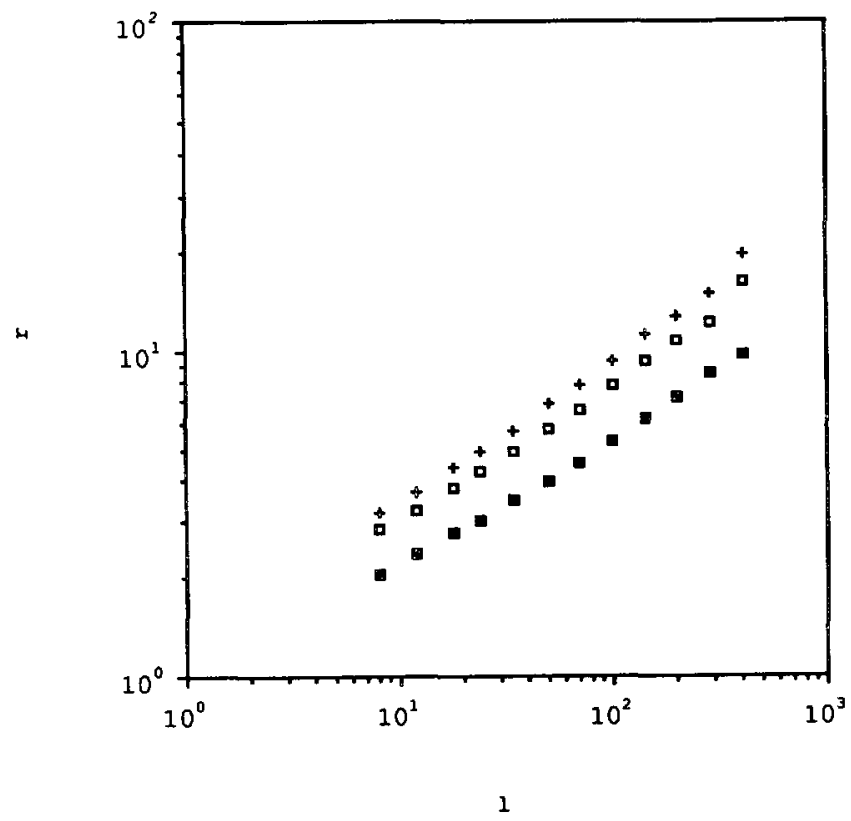

Fig. 11. $-r$ as a function of $\ell$ measured by the largest spread of either closed $(\square)$ or open polymers ( $\square$ ), or as the end-to-end distance for open polymers $(+)$ in two dimensions and a uniform local interaction energy distribution between zero and one (b). 


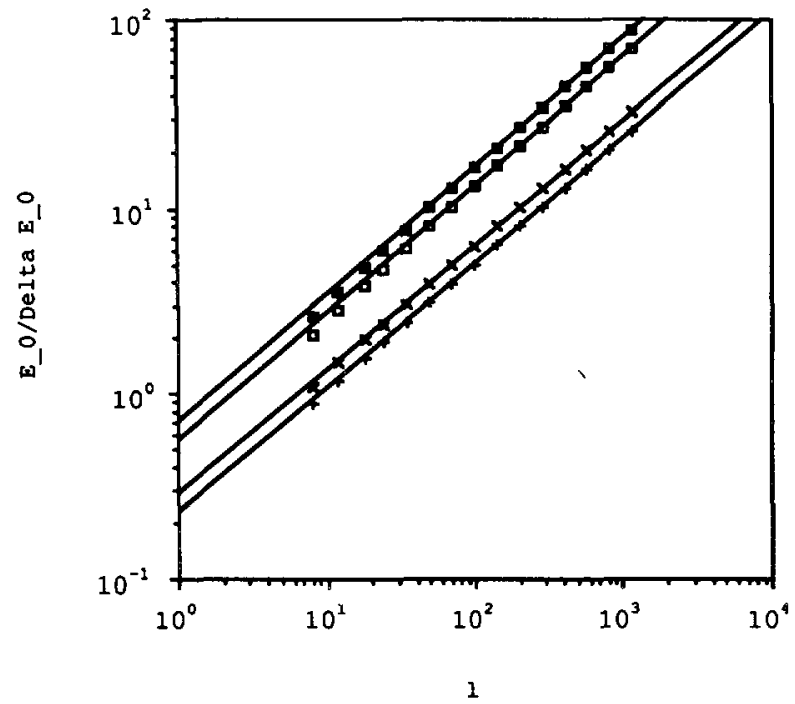

Fig. 12. $-E_{0}$ as a function of $\ell$ for closed ( $\square$ ) and open polymers ( $\square$ ), and the energy fluctuations $\Delta E_{0}$ as a function of $\ell$ for closed $(x)$ and open polymers $(+)$ in one dimension and a uniform local interaction energy distribution between zero and one (b). The straight lines are least-squares fits.

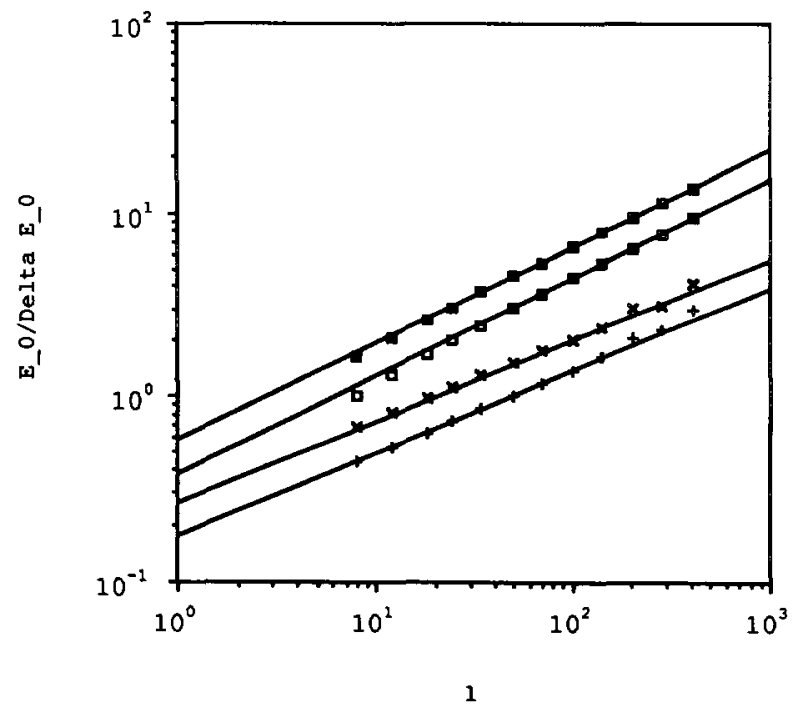

Fig. 13. $-E_{0}$ as a function of $\ell$ for closed $(\square)$ and open polymers ( $\square$ ), and the energy fluctuations $\Delta E_{0}$ as a function of $\ell$ for closed $(x)$ and open polymers $(+)$ in two dimensions and a uniform local interaction energy distribution between zero and one (b). The straight lines are least-squares fits.

for open and closed polymers in one and two dimensions with the local interaction energy distribution (b).

As is evident in table III all the measured exponents $\mu_{\text {meas }}$, governing the scaling of the ground state energy, equation (12), are very close to the exponents governing the energy fluctuations, $\chi_{\text {meas }}$. They are, furthermore, with one exception, less than one. However, as 
was argued following equation (12), $\mu$ should be greater than or equal to one, since the scaling properties of the ground state energy should not be dependent on the addition of a constant term in the local interaction energy. The distributions (a) to (c) have, however, been chosen such that the additional constant is zero, and we see the next-to-leading order scaling in the energy - which is the scaling of the fluctuations, leading to $\mu_{\text {meas }} \approx \chi_{\text {meas }}<1$.

From table III, we see that the scaling exponents as determined numerically are quite close to those predicted by the Flory argument presented in section 2 . Comparing specifically the exponents $\zeta_{\text {meas }}$ and $\zeta_{\text {Flory }}$, we see that the trend is for the measured exponents to be somewhat smaller than those predicted by the Flory theory for the two-dimensional case. A possible explanation of this discrepancy might be the following : in the Flory argument we assumed that the length of the polymer between its starting point and the point at which it curls up, $\ell_{\mathrm{a}}$ is proportional to the distance between the two points, $r$ - see the discussion following equation (7). However, restoring the possibility that $\ell_{\mathrm{a}} \sim r^{\delta}$ with $\delta>1$, leads to a decrease in the corresponding $\zeta$. Equation (10) becomes with this assumption

$$
E(r)=r^{\delta}\langle\eta\rangle_{1}+\left(\ell-r^{\delta}\right) \eta_{\min (1)}
$$

For example assuming that the distribution of local interaction energies is given by a distribution of type (1d), we find that

$$
\zeta=\frac{2 \alpha}{2 \alpha \delta-d}
$$

rather than

$$
\zeta=\frac{2 \alpha}{2 \alpha-d} .
$$

We note that this argument is only valid for $d>1$ - in one dimension the exponent $\delta$ must be one. Returning to table III we see that the difference between $\zeta_{\text {meas. }}$ and $\zeta_{\text {Flory }}$ is less in one than in two dimensions.

We may turn this argument around and use the difference between $\zeta_{\text {meas }}$ and $\zeta_{\text {Flory }}$ in two dimensions to estimate $\delta$. We find a $\delta$ roughly equal to 1.5 for distribution (a) $(\alpha=1 / 2)$ and decreasing for increasing $\alpha$, reaching about 1.2 for $\alpha=2$, distribution (c). First of all, $\delta$ must be between one and two, which turns out to be the case. Secondly, the observed trend that for increasing $\alpha, \delta$ diminishes makes sense, since this implies that the fractal dimension of the path $\mathscr{P}_{\text {a }}$ increases with increasing disorder $(\alpha \rightarrow 0)$. We, however, point out the weakness in this way of estimating $\delta$ : if indeed the path $\mathscr{P}_{a}$ is fractal, there are very strong correlations present, and it is very likely that the Flory approach based on equation (26) will be poor.

\section{Conclusion.}

We have in this paper presented an analytic and numerical study of self-crossing polymers which are strongly interacting with a quenched disordered medium. We presented a complete phase diagram with respect to the disorder of the problem based on a Flory theory. In one dimension the agreement between the numerical results and the Flory argument is perfect. In two dimensions, the agreement is also very good, but differences are detectable.

\section{Acknowledgments.}

We thank H. J. Herrmann for the invitation to HLRZ. This work was supported by the GermanNorwegian Research Cooperation (A.H. and E.L.H.). We also thank one of the referees for interesting remarks and suggestions. 


\section{References}

[1] De Gennes P.-G., Scaling Concepts in Polymer Physics (Cornell University Press, Ithaca, 1979); Doi M., Edwards S. F., The Theory of Polymer Dynamics (Clarendon Press, Oxford, 1986).

[2] Le Doussal P., Machta J., J. Stat. Phys. 64 (1991) 541.

[3] Kardar M., Zhang Y.-C., Phys. Rev'. Lett. 58 (1987) 2087.

[4] Baumgartner A., Muthukumar M., J. Chem. Phys. 87 (1987) 3082.

[5] Edwards S. F., Muthukumar M., J. Chem. Phys. 89 (1988) 2435.

[6] Cates M. E., Ball R. C.. J. Phys. France 49 (1988) 2009.

[7] Nattermann T., Renz W., Phys. Rev. A 40 (1989) 4675.

[8] Wolf D. E., Kertész J., Europhys. Lett. 4 (1987) 651 ;

Halpin-Healy T., Phys. Rev. Lett. 62 (1989) 445 ;

Kim J. M., Kosterlitz J. M., Phys. Rev. Lett 62 (1989) 2289 ;

Halpin-Healy T., Phys. Rev. Lett. 63 (1989) 917 ;

Hentschel H. G. E., Family F.. Phys. Rev. Lett. 66 (1991) 1982.

[9] Zhang Y.-C., J. Phys. France 51 (1990) 2129.

[10] Amar J. G., Family F., J. Phys. A 24 (L) (1991) 295 ;

Roux S., Hansen A., Lucena L. S., Da Silva L. R., Pandey R. B., J. Stat. Phys. 65 (1991) 183.

[11] Roux S.. Hansen A., Guyon E., J. Phys. France 48 (1987) 2125.

[12] Gumbel J., Statistics of Extremes (Columbia University Press, New York, 1958).

[13] Huse D. A., Henley C. L., Phys. Rer. Lett. 54 (1985) 2708.

[14] STAuffer D., Introduction to Percolation Theory (Taylor and Francis, London, 1985). 